\title{
Intestinal microbiota in primary sclerosing cholangitis
}

Johannes R. Hov ${ }^{1,2}$ and Martin Kummen ${ }^{1}$

1. Norwegian PSC Research Center and Research Institute of Internal Medicine, Division of Surgery, Inflammatory Diseases and Transplantation, Oslo University Hospital Rikshospitalet, Oslo; Norway

2. Institute of Clinical Medicine, University of Oslo, Oslo, Norway

\section{Corresponding author:}

Johannes R. Hov, M.D. Ph.D.

Address: Norwegian PSC Research Center, Department of Transplantation Medicine, Oslo University Hospital Rikshospitalet, P.O.Box 4950 Nydalen, 0424 Oslo,

E-mail: j.e.r.hov@medisin.uio.no

Phone: +47 23070000 . 


\begin{abstract}
Purpose of review: Alterations of the gut-liver axis have been linked to the pathogenesis of primary sclerosing cholangitis (PSC) since the disease was first described. The purpose of the review is to discuss multiple recent studies on the intestinal microbiota in human PSC and experimental models of this disease.

Recent findings: Data are available from in total eight cross-sectional studies of human PSC, which include a variable number of patients $(n=11-85)$, material (mucosal or fecal) and microbiota profiling methodology. Despite the heterogeneity of the studies, a pattern of differences is observed that could represent a theme or signature of the PSC gut microbiota, characterized by low diversity and with alterations in multiple bacterial taxa. In experimental models of PSC, re-derivation of animals into germ-free facilities may either aggravate or attenuate the disease, depending on host genetics and putative disease mechanisms (e.g. fibrotic or immune-driven processes, respectively). Summary: The present data provide a strong rationale to explore the functional consequences of the observed gut microbial alterations and their influence on the pathogenesis in PSC. Studies of gut microbiota as biomarker and treatment target may potentially also lead to early translation into clinical practice.
\end{abstract}

\title{
Keywords
}

Primary sclerosing cholangitis, microbiota, metagenomics, metabolomics 


\section{Introduction}

The strong association between primary sclerosing cholangitis (PSC) and inflammatory bowel disease has since the early days suggested that gut microbes could play a key role in the disease process. In fact, antibiotics were the treatment of choice in the first interventional trials in PSC [1], and all common hypotheses of the etiology of the disease may involve the gut microbiota or gutliver axis [2].

The vital role of the commensal gut microbiota as an integrated part of human physiology, through e.g. the production of secondary bile acids, short-chain fatty acids and vitamins, has been established for decades. However, the notion that the gut microbiota (i.e. the microbial contents of the intestine) could be an important contributor to human metabolic and inflammatory diseases started to emerge about a decade ago. Until recently, there was little evidence deriving from PSC or experimental models of the condition. This has lately changed dramatically. The scope of this review is therefore to present an overview of recent studies of the intestinal microbiota in human or experimental of PSC.

\section{Lessons from case-control studies}

Data from at least eight different studies of the gut microbiota in PSC have recently been published as full articles [3-7] or letters to the editor [8-10]. Four of these focused on mucosal biopsies (Table 1) and the last four analyzed fecal samples (Table 2). The studies of the gut mucosal microbiota in PSC are small, including between 11 and 31 PSC patients (Table 1). In the first paper from Rossen et al., a chip-based analysis of the composition of $16 \mathrm{~S}$ rRNA of the samples was performed [3]. The main findings were a reduced intra-individual (alpha) diversity of the mucosal microbiota in PSC $(n=12)$ compared with non-inflamed controls $(n=9)$. In addition, a reduced abundance of uncultured Clostridiales II was observed. The following three studies all used sequencing-based analysis of 
16S rRNA amplicons and except observations of differences between PSC and controls, the results show few signs of overlap (Table 1). An illustration of the challenges in the field was the observation from the study of Canadian and Norwegian patients by Kevans et al. [4], where the main determinant of global microbiota differences was geography, suggesting that multi-center studies are important, but must be performed with great care.

The studies of the fecal microbiota in PSC exhibit more overlap (Table 2). In the largest study from Norway, in total 85 PSC patients were included, using a two-stage design by splitting the participants into a discovery and validation panel [6]. The main findings of this study were that the global microbiota composition was different in PSC patients, compared with healthy controls and ulcerative colitis (UC) patients and characterized by reduced intra-individual diversity. The Veillonella genus was increased in PSC compared with both healthy controls and UC. In addition, using only those taxa most strongly associated with PSC, it was possible to separate PSC from healthy controls with an acceptable area under the curve (0.78).

In a similar study from Belgium by Sabino and Vieira-Silva et al. [7], 66 PSC patients were included, verifying that PSC exhibit reduced intra-individual diversity. An increase in the Veillonella genus was also reported in this study, although this was nonsignificant when removing patients with cirrhosis, suggesting that it may primarily be related to late stage liver disease. In addition, several other taxa were found to be overrepresented in PSC; including Enterococcus, Fusobacterium and Lactobacillus. By using these, cases and controls in the validation panel could be identified with reasonable precision.

The two final studies are so far only published as letters to the editor as a response to the papers mentioned above. In a German cohort with 73 PSC patients, the specific findings by Kummen et al. were reassessed, confirming the increased abundance of Veillonella in PSC compared with controls, 
but not with UC [9]. Applying the selected taxa used to separate PSC and healthy controls in the Norwegian study, they were also able to separate PSC from healthy controls with an AUC of 0.857. Finally, in a study of 13 pediatric PSC patients from Japan, global microbiota differences were seen compared with controls, along with reduced alpha diversity and an increase in Veillonella and Enterococcus [10].

These results represent strong evidence that PSC is associated with an altered gut microbiota. The overlap in the studies of fecal microbiota is almost surprising given the different methods used in all steps of the microbiota analyses, from extraction methods to the choice of target region in the $16 \mathrm{~S}$ rRNA gene. Also, the patient populations were different both in terms of geography, age and exclusion criteria (Table 2). In contrast, the results from the mucosal microbiota studies are more heterogeneous and difficult to interpret. However, one could speculate that the there is an undiscovered functional overlap in all these studies, as they are 16S rRNA based studies investigating microbial composition on taxonomic level. Functional metagenomics studies revealing the genetic content of the gut microbiota have so far not been cost-effective in mucosal samples due to the overwhelming amount of host DNA in these samples. Still, the field should keep studying both fecal and mucosal samples, since these may represent different but important aspects of the gut microbiota in PSC; the luminal microbiota, with its large metabolic capacity, and mucosal microbiota, with its close interaction with host immune cells.

\section{The importance of microbial metabolism and function}

A fundamental feature of the gut microbiota in humans is that the distribution of bacteria present (microbiota profile) is highly variable between individuals, while the microbial functions present (as defined by bacterial genes) are a lot more similar [11]. Determination of the entire metagenome in a disease, i.e. all bacterial genes present, could provide a clue to the functional consequences of the altered microbiota. Only few studies have so far applied large-scale complete metagenomics in 
disease-focused studies [12,13] and none in PSC, as these are expensive and computationally demanding. Microbial functions may also be measured by microbial metabolites in the circulation or feces. The microbial influence on the human metabolome has been proposed as a major pathway for the health effects from the gut. This is best exemplified by the studies on trimethylamine-Noxide (TMAO) in cardiovascular disease $[14,15]$. In several landmark papers it has been shown that microbial metabolism of dietary choline and carnitine to trimethylamine and subsequent oxidation in the liver to TMAO leads to cardiovascular disease, and high levels of TMAO is associated with cardiovascular end-points $[14,15]$. This process is entirely dependent on the gut microbiota and provides a link between diet, microbes and disease.

Metabolomic studies in small numbers of PSC patients have shown that both the global bile acid composition and profile of the circulating metabolome in PSC differs from controls [16,17]. This may in part be caused by altered microbial functions, but whether it has an impact on disease development is not known. In a recent Norwegian study, the concentration of the microbiota dependent metabolite TMAO was lower in PSC patients $(n=305)$ than in controls [18]. When removing patients with reduced liver function, the concentrations were similar, suggesting that the final step of TMAO synthesis in the liver is impaired in late stage disease. In the subgroup with normal liver function elevated TMAO was associated with reduced liver transplantation-free survival, suggesting that the interaction of diet and gut microbial function may be relevant for disease progression, although the study is limited by the lack of data on diet and antibiotics use at sampling. Importantly, in PSC, high TMAO could be speculated to involve elevated levels of the precursor trimethylamine, since experimental studies suggest that TMAO and related methylamines induce cholestasis, cholangiocyte proliferation and cholangiofibrosis [19], with increased trimethylamine in males [20]. Of note, both Veillonella species and several bacteria reported to be enriched in the mucosa of PSC patients contain genes that encode amine oxidases producing primary amines [8]. 


\section{Genetic and environmental determinants of the host microbiota}

Twin studies suggest that the microbiota is in part heritable [21]. Extending from the genetic studies in inflammatory diseases like PSC, there has been an increasing interest to investigate if disease susceptibility genes influence the microbiota. However, using a unique selection of healthy individuals being homozygote for the strongest genetic risk factor in PSC, the HLA-B*08:01DRB1*03:01 haplotype, only very modest associations with gut microbiota composition were observed, suggesting that the effects are very small or that this haplotype does not increase disease susceptibility via the gut [22]. In contrast, in IBD, a genetic risk score based on susceptibility genes involved in bacterial handling was associated with gut microbiota profile in healthy individuals [23], suggesting that IBD risk genes influence gut microbial composition.

The concept of gene-microbiota interaction has also been investigated in large population-level cohorts [24-26]. The results provide compelling evidence that the gut microbiota composition is in part dependent on variation in host genetics. On a group level, the effects of genetic variation were as strong as the effects of non-genetic factors (e.g. diet, body-mass index, gender and smoking) [24]. Of particular interest is the identification of genetic variation in the vitamin D receptor as a determinant of the gut bacterial composition and bacterial bile acid-related gene pathways [24]. Since the vitamin D receptor is an important receptor for microbiota transformed secondary bile acids like lithocholic acid, in addition to vitamin D [27], these data identify important hostmicrobial interactions, which may be highly relevant for bile acid homeostasis and the biliary environment.

\section{The gut microbiota in animal models of PSC}

In order to understand the causal relationship between gut microbes and disease, gnotobiotic or germ-free animals are important tools. Recently the role of the gut microbiota has been investigated 
in two mouse models of PSC [28,29]. Multidrug resistance gene 2 knockout $\left(M d r 2^{-/}\right)$mice lack phospholipids in the bile and acquire a sclerosing cholangitis characterized by fibrosis, which is widely used as a mouse model for PSC. In an experiment conducted by Tabibian et al., $M d r 2^{-/-}$mice were re-derived into and maintained at a germ-free facility [28]. These mice exhibited exaggerated liver disease as shown by increased cholestasis and fibrosis, ductopenia and ductular reaction, as well as increased cholangiocyte senescence. As expected, circulating secondary bile acids, which are entirely dependent on microbial metabolism, were completely absent in the germ-free mice. This may have caused the worsened phenotype. In contrast, in a study by Schrumpf and Kummen et al. using the NOD.c3c4 mouse model of immune-driven biliary inflammation, re-derivation of the mice into a germ-free facility attenuated the liver disease [29]. Similar but weaker effects were seen in mice receiving non-absorbable antibiotics from weaning, while co-housing experiments were unable to detect a transmissible phenotype. Also, NOD.c3c4 mice had different gut microbiota composition than control mice, even after re-derivation into a new animal facility.

These two studies are of particular interest showing that an altered gut microbiota have opposite effects depending on the nature of the disease process and host genes. Other models of liver disease characterized by fibrosis (a hallmark of disease in $M d r 2^{-/}$mice) are also worsened under germ-free conditions [30]. Germ-free models can be criticized for being highly unnatural, with considerable changes in their immune system, and consequently it would be interesting to investigate both whether $M d r 2^{-/}$mice treated with antibiotics also exhibit worsening of their liver disease, and whether this phenotype could be transmissible in co-housing experiments. Also, based on the available data, colonization experiments using e.g. Enterococcus or Veillonella would be of interest in relevant models.

\section{Discussion and the road ahead}

Two main conclusions can be drawn with reasonable certainty from present studies of the intestinal microbiota in PSC: 1) PSC patients have an altered gut microbiota characterized by reduced 
diversity and alterations of specific taxa and 2) Gut microbiota alterations in mouse models of chronic cholangitis may improve or worsen the disease phenotype depending on the host genes and the driving mechanisms of disease. Whether, or how, these two observations are linked is not known, and it is probably too early to conclude that the observed microbial alterations in human PSC are relevant for the pathogenesis or could have implications for clinical practice. Still, the observations provide an important starting point and strong rationale to prioritize this field ahead. The limitations of the present data include the cross-sectional design, the selection of controls and the multiple biases associated with PCR-based microbiota profiling. Importantly, the effect size of gut microbiota alterations may be small, perhaps in the range of what has been observed in the genetics of common diseases. The current studies are therefore probably highly underpowered and insensitive to microbial alterations that could be of importance, urging the community to collaborate to increase study size. Importantly, a definition of a "pathogenic" microbiota does not exist, and therefore we do not know what to look for. Is disease caused, or altered in its development, by e.g. a "dysbiotic ecosystem" or a single pathogenic microbe? Increasing evidence suggest that microbial functions are more important than the microbial composition. It will therefore be important to delineate functional consequences of the observed microbiota alterations by applying complete metagenomics to study gene content and metabolomics to capture the fingerprints of microbial metabolism.

The published data are promising since a microbial signature of PSC may exist. This means that the gut microbiota may be of clinical importance long before causality is understood or established $[31,32]$. The individual differences in gut microbiota profile, and the excellent ability of simple profiles to separate different phenotypes suggest that the gut microbiota is relevant as a biomarker [31]. Biomarkers could be diagnostic, but it would be clinically more relevant to assess the value of the gut microbiota as biomarker of prognosis. Such associations would also strengthen the link between gut microbiota and the disease process in PSC. Targeting the microbiota in treatment trials 
in human disease could also provide strong proof for a mechanistic role of the gut microbiota. Several trials of antibiotics have been performed in PSC [33,34], and in particular pilot trials of vancomycin show some promise. Fecal microbiota transplantation (FMT) could also be tested. Two recent studies of FMT in UC have provided some lessons on the potential and pitfalls of this approach $[35,36]$. There were signs of effect, with one of the studies reaching the primary endpoint [36]. However, issues like choice of donors, administration route and frequency of FMT need further study. In addition, data also suggest that to achieve remission of colitis, treatment early in disease course may be critical, and this could be a challenge in PSC [36].

\section{Conclusion}

Data from multiple studies have now firmly established the presence of an altered composition of gut bacteria in PSC. Further work needs to focus on defining the details of this in larger studies, as well as characterizing the functional consequences, and include viral and fungal contributions to the microbiota. In addition, we are still awaiting the definite proof that gut microbiota alterations have pathogenetic or clinical impact in human PSC, which could be provided by experimental or proofof-concept clinical trials.

\section{Key points:}

- Multiple studies have now firmly established the presence of an altered gut microbial composition in PSC.

- The gut microbiota in PSC is characterized by low diversity and alterations in multiple bacterial taxa.

- Experimental models of PSC show aggravated or attenuated disease when re-derived into germ-free facilities, probably depending on host genotype and the dominating disease mechanisms in the models. 
- Further studies with larger cohorts and a focus on functional bacterial alterations will be important, in addition basic translation to experimental studies.

- Proof-of-concept clinical studies of the gut microbiota as biomarker or treatment target may potentially lead to altered clinical practice before the mechanisms are fully elucidated

\section{Financial support and sponsorship}

JRH is funded by the Norwegian Research Council (grant 240787/F20). MK is funded by the Regional Health Authority of South-Eastern Norway (project number 2016067).

\section{Conflicts of interest}

None. 


\section{References}

1. Rankin JG, Boden RW, Goulston SJ, Morrow W: The liver in ulcerative colitis; treatment of pericholangitis with tetracycline. Lancet 1959, 2:1110-1112.

2. Karlsen TH, Schrumpf E, Boberg KM: Update on primary sclerosing cholangitis. Dig Liver Dis 2010, 42:390-400.

3. Rossen NG, Fuentes S, Boonstra K, et al.: The mucosa-associated microbiota of PSC patients is characterized by low diversity and low abundance of uncultured Clostridiales II. $J$ Crohns Colitis 2015, 9:342-348.

*First study of the gut microbiota in PSC detecting a reduced intra-individual bacterial diversity in the mucosa-adherent microbiota. A single cohort was investigated with a probe-based analysis of 16S rRNA sequences.

4. Kevans D, Tyler AD, Holm K, et al.: Characterization of Intestinal Microbiota in Ulcerative Colitis Patients with and without Primary Sclerosing Cholangitis. J Crohns Colitis 2016, 10:330-337.

5. Torres J, Bao X, Goel A, et al.: The features of mucosa-associated microbiota in primary sclerosing cholangitis. Aliment Pharmacol Ther 2016, 43:790-801.

*Largest study of the mucosal microbiota in PSC to date. Identified bacterial taxa involved in bile acids metabolism as enriched in PSC patients.

6. Kummen M, Holm K, Anmarkrud JA, et al.: The gut microbial profile in patients with primary sclerosing cholangitis is distinct from patients with ulcerative colitis without biliary disease and healthy controls. Gut 2016. doi: 10.1136/gutjnl-2015-310500

*First and largest study of fecal gut microbiota in PSC, identifying a low diversity microbiota profile with Veillonella being a PSC associated genus. Microbiota profile was suggested to have diagnostic abilities. 
7. Sabino J, Vieira-Silva S, Machiels K, et al.: Primary sclerosing cholangitis is characterised by intestinal dysbiosis independent from IBD. Gut 2016, 65:1681-1689.

*Large study of the fecal gut microbiota, showing a low diversity microbiota profile with elevated Enterococcus, Fusobacterium and Lactobacillus, which together had the ability to discriminate between case and control.

8. Quraishi MN, Sergeant M, Kay G, et al.: The gut-adherent microbiota of PSC-IBD is distinct to that of IBD. Gut 2016. doi: 10.1136/gutjnl-2016-311915

9. Ruhlemann MC, Heinsen FA, Zenouzi R, et al.: Faecal microbiota profiles as diagnostic biomarkers in primary sclerosing cholangitis. Gut 2016. doi: 10.1136/gutjnl-2016-312180

10. Iwasawa K, Suda W, Tsunoda T, et al.: Characterisation of the faecal microbiota in Japanese patients with paediatric-onset primary sclerosing cholangitis. Gut 2016. doi:10.1136/gutjnl2016-312533

11. The Human Microbiome Project Consortium: Structure, function and diversity of the healthy human microbiome. Nature 2012, 486:207-214.

12. Qin J, Li Y, Cai Z, et al.: A metagenome-wide association study of gut microbiota in type 2 diabetes. Nature 2012, 490:55-60.

13. Qin N, Yang F, Li A, et al.: Alterations of the human gut microbiome in liver cirrhosis. Nature 2014.

14. Wang Z, Klipfell E, Bennett BJ, et al.: Gut flora metabolism of phosphatidylcholine promotes cardiovascular disease. Nature 2011, 472:57-63.

15. Tang WH, Wang Z, Levison BS, et al.: Intestinal microbial metabolism of phosphatidylcholine and cardiovascular risk. N Engl J Med 2013, 368:1575-1584.

16. Bell LN, Wulff J, Comerford M, et al.: Serum metabolic signatures of primary biliary cirrhosis and primary sclerosing cholangitis. Liver Int 2015, 35:263-274. 
17. Trottier J, Bialek A, Caron P, et al.: Metabolomic profiling of 17 bile acids in serum from patients with primary biliary cirrhosis and primary sclerosing cholangitis: a pilot study. Dig Liver Dis 2012, 44:303-310.

18. Kummen M, Vesterhus M, Troseid M, et al.: Elevated trimethylamine-N-oxide (TMAO) is associated with poor prognosis in primary sclerosing cholangitis patients with normal liver function. United European Gastroenterol J 2016.

19. Lin JK, Ho YS: Hepatotoxicity and hepatocarcinogenicity in rats fed squid with or without exogenous nitrite. Food Chem Toxicol 1992, 30:695-702.

20. Bennett BJ, de Aguiar Vallim TQ, Wang Z, et al.: Trimethylamine-N-oxide, a metabolite associated with atherosclerosis, exhibits complex genetic and dietary regulation. Cell Metab 2013, 17:49-60.

21. Goodrich JK, Waters JL, Poole AC, et al.: Human genetics shape the gut microbiome. Cell 2014, 159:789-799.

22. Hov JR, Zhong H, Qin B, et al.: The Influence of the Autoimmunity-Associated Ancestral HLA Haplotype AH8.1 on the Human Gut Microbiota: A Cross-Sectional Study. PLoS One 2015, 10:e133804.

23. Imhann F, Vich Vila A, Bonder MJ, et al.: Interplay of host genetics and gut microbiota underlying the onset and clinical presentation of inflammatory bowel disease. Gut 2016.

24. Wang J, Thingholm LB, Skieceviciene J, et al.: Genome-wide association analysis identifies variation in vitamin D receptor and other host factors influencing the gut microbiota. Nat Genet 2016, 48:1396-1406.

**German-International study reporting on the role of genetics on the microbiota in two human population-based cohorts, identifying the vitamin D receptor gene with a possible key role in regulation of gut bacterial composition, with further associations with both bile- and fatty-acid profiles. 
25. Turpin W, Espin-Garcia O, Xu W, et al.: Association of host genome with intestinal microbial composition in a large healthy cohort. Nat Genet 2016, 48:1413-1417.

*Large Canadian study of gene-microbiota interactions identifying an association between a single nucleotide polymorphism (SNP) on chromosome 2 and Rikenellaceae abundance.

26. Bonder MJ, Kurilshikov A, Tigchelaar EF, et al.: The effect of host genetics on the gut microbiome. Nat Genet 2016, 48:1407-1412.

*Dutch study exploring the influence of human genetics on the microbiota by metagenomics sequencing, identifying gene-diet interacions as important for regulation of Bifidobacterium abundance.

27. Makishima M, Lu TT, Xie W, et al.: Vitamin D receptor as an intestinal bile acid sensor. Science 2002, 296:1313-1316.

28. Tabibian JH, O'Hara SP, Trussoni CE, et al.: Absence of the intestinal microbiota exacerbates hepatobiliary disease in a murine model of primary sclerosing cholangitis in mice. Hepatology 2016;63:185-96

**First study of a mouse model of biliary disease in a germ-free state. Surprisingly, germ-free mice exhibited exaggerated disease compared with normal mice. A lack of microbial transformation of bile acids or other microbial metabolites was proposed as the cause of this intriguing observation.

29. Schrumpf E, Kummen M, Valestrand L, et al.: The gut microbiota contributes to a mouse model of spontaneous bile duct inflammation. J Hepatol 2016. doi:

10.1016/j.jhep.2016.09.020

*In an immune-driven mouse model of biliary disease, the biliary disease seemed to drive alterations in gut microbial profile. Re-derivation into a germ-free state attenuated the liver disease, providing a highly interesting contrast to ref 28 , which investigated a model with a stronger link to fibrosis.

30. Mazagova M, Wang L, Anfora AT, et al.: Commensal microbiota is hepatoprotective and prevents liver fibrosis in mice. FASEB J 2015, 29:1043-1055. 
31. Raes J: Microbiome-based companion diagnostics: no longer science fiction? Gut 2016, 65:896-897.

32. Knight R: Why microbiome treatments could pay off soon. Nature 2015, 518:S5.

33. Tabibian JH, Weeding E, Jorgensen RA, et al.: Randomised clinical trial: vancomycin or metronidazole in patients with primary sclerosing cholangitis - a pilot study. Aliment Pharmacol Ther 2013, 37:604-612.

34. Farkkila M, Karvonen AL, Nurmi H, et al.: Metronidazole and ursodeoxycholic acid for primary sclerosing cholangitis: a randomized placebo-controlled trial. Hepatology 2004, 40:1379-1386.

35. Rossen NG, Fuentes S, van der Spek MJ, et al.: Findings From a Randomized Controlled Trial of Fecal Transplantation for Patients With Ulcerative Colitis. Gastroenterology 2015, 149:110-118 e114.

36. Moayyedi P, Surette MG, Kim PT, et al.: Fecal Microbiota Transplantation Induces Remission in Patients With Active Ulcerative Colitis in a Randomized Controlled Trial. Gastroenterology 2015, 149:102-109 e106. 